\title{
RECONOCIMIENTO DE LA HOMOSEXUALIDAD, APOYO \\ SOCIAL, DEPRESIÓN E IDEACIÓN SUICIDA EN PERSONAS \\ HOMOSEXUALES
}

\section{Recognition of Homosexuality, Social Support, Depression and Suicidal Ideation in Homosexual People}

\author{
Ana Karen Ceballos Mora ${ }^{1}$, Christian Alexander Zambrano \\ Guerrero², Fredy Hernán Villalobos Galvis ${ }^{3}$, Sara Natalia \\ Guerrero Caicedo ${ }^{4}$
}

doi: https://doi.org/10.17533/udea.rp.e342120

\section{Resumen}

La investigación tuvo como propósito determinar la relación entre apoyo social, depresión y reconocimiento de la homosexualidad, con la ideación suicida en personas homosexuales de San Juan de Pasto, Colombia. El tipo de estudio fue correlacional, con una muestra no probabilística por conveniencia de 162 personas que se autodefinían como homosexuales. Se aplicó las escalas de Apoyo Social, de Depresión del Centro de Estudios Epidemiológicos, de Reconocimiento de la Homosexualidad, así como el Inventario de Ideas Suicidas Positivas y Negativas. Se encontró que los participantes presentaron mayores niveles de ideación suicida y depresión, y menores niveles de apoyo social, en comparación con datos obtenidos en la población general de San Juan de Pasto. Se concluye que la depresión está asociada directamente con la ideación suicida, mientras que el apoyo social y el reconocimiento de la orientación sexual promueven la salud mental en gais y lesbianas.

Palabras clave: reconocimiento, homosexualidad, ideación suicida, apoyo social, depresión.
Recibido: 2020-05-19 / Aceptado: 2020-12-07

Para citar este artículo en APA: Ceballos, A., Zambrano, C., Villalobos, F. y Guerrero, S. (2021). Reconocimiento de la homosexualidad, apoyo social, depresión e ideación suicida en personas homosexuales. Revista de Psicología Universidad de Antioquia, 13(1), e342120. doi: https://doi. org/10.17533/udea.rp.e342120
Magister en Salud Pública, Universidad de Narińo. Correo: ceballosank@udenar.edu.co; http://orcid.org/0000-0002-4980-4056.

2 Doctorando en Ciencias de la Educación, Universidad de Nariño; https://orcid.org/00000001-8482-0345.

3 Doctor en Psicología Clínica y de la Salud, Universidad de Nariño; https://orcid.org/00000002-2602-0283.

4 Especialista en Organizaciones, Responsabilidad Social y Desarrollo, Universidad de Nariño; https://orcid.org/0000-0002-1425-0970. 
[2] Ana Karen Ceballos Mora, Christian Alexander Zambrano Guerrero, Fredy Hernán Villalobos Galvis, Sara Natalia Guerrero Caicedo

\begin{abstract}
The purpose of the research was to determine the relationship between social support, depression, and recognition of homosexuality, with suicidal ideation in homosexual people in San Juan de Pasto (Colombia). The type of study was correlational, with a non-probabilistic convenience sample of 162 people who defined themselves as homosexuals. The Social Support Scale, the Depression Scale of the Center for Epidemiological Studies, the Homosexuality Recognition Scale as well as the Inventory of Positive and Negative Suicidal
\end{abstract}

Ideas were applied. Participants were found to have higher levels of suicidal ideation and depression, and lower levels of social support, compared to data obtained in the general population of San Juan de Pasto. It concludes that depression is directly associated with suicidal ideation, while social support and recognition of sexual orientation promote mental health in gays and lesbians.

Keywords: recognition, homosexuality, suicide ideation, social support, depression.

\title{
Introducción
}

El suicidio fue definido por Durkheim como aquellos comportamientos directos e indirectos que realiza una persona contra sí misma para producirse la muerte (Jasso, 2013), independientemente del método y del resultado obtenido (Peña, Ortiz y Gutiérrez, 2009). En el suicidio intervienen causas biológicas, culturales, psicológicas, económicas y sociales, entre otras (Benavides, Villota y Villalobos, 2019); es la consecuencia de una dificultad en la adaptación de la persona, relacionada con los recursos y valores que se establecen principalmente desde la familia, y generada por conflictos actuales o permanentes, excesivamente aversivos para el sujeto, lo que no le permite analizar objetivamente la situación, ni formular alternativas diferentes al suicidio (Arláez, Hernández, Álvarez y Cañizares, 1998; Cortina, Peña y Goméz, 2009; Ojeda y Villalobos, 2011; Chaparro, Díaz y Castañeda, 2019).

En el fenómeno del suicidio se identifican tanto los pensamientos como el acto suicida en sí, además, el suicidio consumado, los intentos previos fallidos y los intentos de baja letalidad (Gutiérrez, Contreras y Orozco, 2006). Por su parte, la ideación abarca los pensamientos asociados con causarse daño físico o la muerte, que oscilan desde la desvalorización de la vida, hasta la planeación de la muerte, por lo cual las ideas suicidas han sido consideradas como un factor de riesgo para el suicidio y su estudio se ha convertido en una forma de diagnóstico y de prevención de dicho acto (Muñoz et al., 2006; Villalobos, 2009; Vírseda et al., 2011). 
De acuerdo con la Organización Mundial de la Salud [oms], alrededor de 800000 individuos se suicidan al ańo, lo que significa una muerte aproximadamente cada 40 segundos. El suicidio es la segunda causa de muertes en los jóvenes de 15 a 29 años (oms, 2018). Diversos estudios han documentado la asociación existente entre las conductas suicidas y problemáticas tales como: trastornos depresivos, consumo de sustancias psicoactivas, aumento en los estresores sociales, dificultades psicosociales, historia familiar de suicidio, pérdidas, discriminación, abuso sexual y crisis económicas, entre otras (Peña, Ortiz y Gutiérrez, 2009; Siabato y Salamanca, 2015; Tomicic et al., 2016; Villalobos, Ojeda y Luna, 2019).

Otros estudios refieren que grupos marginados (p.e. homosexuales) son más vulnerables a tener episodios depresivos, los cuales pueden conducir al suicidio (Plderl, Faistauer y Fartacek, 2010). De esta manera, en comparación con las personas heterosexuales, las personas homosexuales muestran mayores probabilidades (de 2 a 6 veces) de suicidarse y mayores tasas de problemas mentales como estrés, ansiedad, depresión y ataques de pánico, particularmente entre adolescentes en el periodo de reconocimiento, aceptación y develación de su orientación sexual ante la sociedad (Granados y Delgado, 2008; Li, Pollitt y Russell, 2015; Barros Dos Santos et al., 2017).

La mayoría de los estudios realizados con población LGBTi (lesbianas, gais, bisexuales, transgénero e intersexual) establecen una mayor frecuencia de ideas y comportamientos suicidas en las personas homosexuales, unido a un número de tentativas de 5 a 10 veces superior que las de la población general (Ortiz y García, 2005). Un estudio en Minnesota con jóvenes homosexuales señala que el $30 \%$ había realizado alguna vez un intento de suicidio, y el 4,7\% lo había intentado en el último año, mostrando un porcentaje superior al de la población general (Schlatter, Irala y Escamilla, 2005).

Asimismo, Bagley y Tremblay (1997) indican que la probabilidad de suicidio en hombres gay y bisexuales se triplicó de 1950 a 1990 en Estados Unidos. Otro estudio encontró que personas jóvenes homosexuales tienen siete veces más probabilidad de cometer intentos de suicidio en comparación con personas heterosexuales de similares características demográficas (Madrigal y Suárez, 2000). 
[4] Ana Karen Ceballos Mora, Christian Alexander Zambrano Guerrero, Fredy Hernán Villalobos Galvis, Sara Natalia Guerrero Caicedo

Langhinrichsen, Lamis y Malone (2010), al analizar los datos de vida social de lesbianas que viven en el sur de Estados Unidos, encontraron que más del $40 \%$ de las participantes había considerado seriamente el suicidio y más del 15\% había intentado suicidarse. Dentro de los predictores de la ideación y los intentos suicidas se incluyeron: síntomas depresivos, la discriminación, el apoyo social, la autoestima y el estigma.

Ortiz y García (2005) analizaron las tasas de ideación e intento suicida en homosexuales y bisexuales de Ciudad de México y señalaron que el 39\% de esta población presenta ideación y el 15\% ha tenido algún intento; mientras que en la población urbana de México se encuentran prevalencias de ideación de $6.3 \%$ a $18.2 \%$ y de intento de $0.9 \%$ a $2 \%$. En los adolescentes LGB (menores de 19 años) las prevalencias de ideación e intento son de $60.3 \%$ y $26.1 \%$, respectivamente, mientras que en los adultos LGB (de 19 años y más) las tasas fueron de $35.7 \%$ y $13.7 \%$.

En conclusión, hay un mayor riesgo de suicidio en las minorías sexuales que en la población heterosexual (Cochran y Mays, 2009; Haas et al., 2011; Barros Dos Santos et al., 2017). Al respecto, los resultados obtenidos por Mereish, O'Cleirigh y Bradford (2013) sugieren que los hombres homosexuales y bisexuales tienen cuatro veces más probabilidades de intentar suicidarse durante su vida que los hombres heterosexuales, mientras que las mujeres lesbianas y bisexuales tienen dos veces más probabilidad de intentar suicidarse que las heterosexuales. Sin embargo, el suicidio no puede asumirse como un resultado directo de la orientación sexual, pero esta es considerada como una posible fuente de circunstancias estresantes para los individuos, relacionadas con discriminación, estigma, violencia, falta de aceptación y aislamiento, lo que, a su vez, incrementa el riesgo de comportamientos suicidas (Irwin y Austin, 2013).

Entre los agentes estresores que están fuertemente asociados con el riesgo de suicidio en personas homosexuales o bisexuales, se destacan los referentes al prejuicio y la homofobia, tales como el limitado apoyo familiar, la exclusión social, la invisibilización, el bullying y la victimización —que además conllevan la posibilidad de mayor aislamiento, rechazo familiar, consumo de sustancias psicoactivas y escasas relaciones interpersonales- (Chomali, 2008; New- 
comb et al., 2014; Stone et al., 2014). En contraste, un factor que promueve el bienestar de gais y lesbianas es el reconocimiento de su homosexualidad, proceso que "involucra una serie de etapas que van desde el surgimiento hasta la consolidación de la persona como ser homopensante, homodeseante, homoafectivo, homoerótico y homogenital" (Zambrano, Hernández y Guerrero, 2019, p. 5), lo cual permite mayor aceptación de sí mismo, autoconfianza y desarrollo de las capacidades personales y sociales del individuo (Zambrano, Ceballos y Ojeda, 2017).

El presente estudio buscó determinar la correlación entre ideación suicida, apoyo social, depresión y reconocimiento de la homosexualidad, en un grupo de personas homosexuales de la ciudad de San Juan de Pasto, Colombia.

\section{Método}

\section{Participantes}

Se implementó un muestreo por conveniencia debido a la dificultad que implica obtener una muestra aleatoria de homosexuales. Participaron 162 personas que se autodefinían como gay $(\mathrm{n}=114)$ o lesbianas $(\mathrm{n}=48)$, con edades entre 18 y 68 años (media de 26 años), y residentes en la ciudad de San Juan de Pasto.

El 70\% de los participantes eran solteros, el 26\% vivían en unión libre y el $3.6 \%$ se encontraban en otro estado civil. Además, el 3\% tenía estudios primarios, $10 \%$ secundarios, $14 \%$ técnicos, $8.6 \%$ tecnológicos y $64.2 \%$ universitarios; $y$, finalmente, el $64.8 \%$ de los participantes pertenecían a fundaciones LGBTI+.

\section{Procedimiento}

Para llevar a cabo este estudio, se invitó a personas homosexuales, a través de fundaciones LGBTI+ y de muestreo de bola de nieve. Una vez convocado el grupo, se procedió a darle información pertinente sobre la investigación y a aclarar el carácter voluntario del estudio. Se hizo entrega del formato de con- 
[6] Ana Karen Ceballos Mora, Christian Alexander Zambrano Guerrero, Fredy Hernán Villalobos Galvis, Sara Natalia Guerrero Caicedo

sentimiento informado y solo a quienes lo firmaron les fueron aplicadas las escalas. Los datos se sistematizaron y analizaron con el programa spss versión 25. Dado que la selección de la muestra no fue aleatoria y que su tamaño no es representativo de la población, y que teóricamente no se espera que las variables presenten una distribución normal, se optó por el uso del Coeficiente de Correlación Rho $(\rho)$ de Spearman, una alternativa no paramétrica al coeficiente de Pearson (Restrepo y González, 2007). De igual manera, se utilizó el software G*Power versión 3.1.9.2 (Faul, Erdfelder, Lang y Buchner, 2007) para calcular la potencia estadística (P) post-hoc de cada $\rho$, en función del tamaño de muestra y el error $\alpha$. Teniendo en cuenta que el tamaño del efecto (w) para la correlación se entiende como $|\rho-\rho 0|$ (G*Power 3.1 manual, 2020) y que la hipótesis nula en este estudio es $\rho 0=0$, el valor de $w=\rho$. Para la interpretación de w se asumieron los criterios mencionados por Iraurgi (2009).

\section{Instrumentos}

Los instrumentos utilizados para el proceso de recolección de datos fueron:

Escala de Apoyo Social - AS (Matud, 1998). Evalúa la percepción global del apoyo social. La versión utilizada (Villalobos, 2006), se compone de 13 ítems que recogen información sobre la percepción de disponibilidad de las personas que pueden prestar apoyo emocional en áreas personales, laborales y familiares. Este instrumento presenta validez de constructo, pues todos los ítems se agrupan en un solo factor y su alfa de Cronbach es mayor de 0.8 (Matud et al., 2002; Villalobos, 2006). En la muestra participante se obtuvo un alfa de Cronbach 0.959 .

Escala de Depresión del Centro de Estudios Epidemiológico (CES-D-R). Diseñada para medir síntomas depresivos en la población en general, se estructura en 4 factores: afecto depresivo, aspectos sociales, sintomas somáticos y afecto positivo. Se utilizó la versión adaptada por González y Andrade (1995) y validada por Villalobos (2010a) en población de San Juan de Pasto, en donde presentó validez de constructo y un alfa de Cronbach de 0.877 . En este estudio se obtuvo un alfa de Cronbach 0.945.

Escala de Reconocimiento de la Homosexualidad (RH). Fue diseñada para la presente investigación e incluye el reconocimiento personal y social de la 
orientación sexual homosexual. Se realizó el proceso de construcción psicométrica del instrumento y se evaluó su validez de contenido y de constructo. Para los datos de este estudio se obtuvo un alfa de Cronbach de 0.95 para la escala total y mayores a 0.9 para las subescalas.

Inventario de Ideas Suicidas Positivas y Negativas (PANSI). Está compuesto por dos factores: la ideación suicida negativa (factores de riesgo) y la ideación suicida positiva (factores protectores), evaluadas en las últimas dos semanas. La ideación suicida positiva se califica de manera inversa, para dar una puntuación directa de la cognición autolítica. El pansi fue creado por Osman et al. (1998) y adaptado a la población pastusa por Villalobos (2010b), presentando una adecuada fiabilidad, con un alfa de Cronbach de 0.899 , y validez de constructo. En el presente estudio se obtuvo un alfa de Cronbach de 0.92.

\section{Consideraciones éticas}

El estudio se desarrolló teniendo en cuenta los principios éticos de justicia, libertad, beneficencia y no maleficencia que permiten salvaguardar los derechos y bienestar de los participantes. Para la realización del estudio se contó con la autorización del Comité de Ética de la universidad financiadora, además de obtener el consentimiento informado de los participantes. El trabajo de campo fue realizado y supervisado por profesionales con conocimiento y experiencia para cuidar la integridad de los participantes y garantizar su bienestar.

\section{Resultados}

En primer lugar, se compararon las puntuaciones de ideas suicidas, depresión y apoyo social de los participantes, con las reportadas en población de bachillerato en la ciudad de San Juan de Pasto (Villalobos, 2006), hallándose que los homosexuales tienen mayores valores de ideación suicida total, ideación suicida negativa y depresión, así como menores valores de apoyo social (ver Tabla 1). 
[8] Ana Karen Ceballos Mora, Christian Alexander Zambrano Guerrero, Fredy Hernán Villalobos Galvis, Sara Natalia Guerrero Caicedo

Tabla 1.

Comparación de ideación suicida entre la muestra de estudio y población de referencia

\begin{tabular}{|c|c|c|c|c|c|c|}
\hline Muestra & & PANSI & IS & IS & CES-D-R & AS \\
\hline & & & Positivas & Negativas & & \\
\hline Participantes & Media & 12.96 & 7.51 & 5.46 & 46.67 & 22.14 \\
\hline & D.E. & 10.438 & 5.52 & 6.46 & 12.006 & 14.646 \\
\hline & $\mathrm{N}$ & 162 & 162 & 162 & 162 & 162 \\
\hline Población & Media & 10.85 & 7,64 & 3.22 & 20.21 & 36.44 \\
\hline Referencia & D.E. & 9.85 & 5.62 & 5.96 & 13.44 & 11.11 \\
\hline & $\mathrm{N}$ & 357 & 357 & 357 & 345 & 369 \\
\hline Prueba de & z & 2.171 & 0.247 & 3.748 & 22.257 & -11.104 \\
\hline Hipótesis & p-valor & .015 & 0.402 & $<.001$ & $<.001$ & $<.001$ \\
\hline
\end{tabular}

En segundo lugar, se analizó la correlación entre las puntuaciones de las pruebas y se encontraron asociaciones estadísticamente significativas entre las variables de estudio: correlación directa y alta entre As y RH $(\rho=0.529$; $\mathrm{p}<0,001 ; \mathrm{P}=0.999)$ y correlaciones inversas y altas entre CES-D-R y AS $(\rho$ $=-0.664 ; \mathrm{p}<0,001 ; \mathrm{P}=1)$ y entre CES-D-R y RH $(\rho=-0.503 ; \mathrm{p}<0,001 ; \mathrm{P}=$ $0.999)$, resultados que corroboran cómo la depresión tiene una asociación inversa con las otras dos variables de estudio y estas, a su vez, muestran una asociación positiva entre ellas. Se validó el uso del coeficiente $\rho$ al hallarse que las variables de estudio presentaban asimetrías (g1) o curtosis (g2) diferentes a cero: apoyo social $(\mathrm{g} 1=-.249$ y g2 $=-.873)$, depresión $(\mathrm{g} 1=.643$ y g2 = $.114)$, reconocimiento social $(\mathrm{g} 1=.050$ y g2 $=-1.047)$ e ideación suicida ( $\mathrm{g} 1$ $=.535$ y g2 $=-.924)$.

Por otro lado, las asociaciones entre los correlatos (AS, CES-D-R y RH) y la ideación suicida se evidencian en coeficientes de correlación significativos y, en la gran mayoría, con tamaño del efecto mayor al típico $(\rho>0,5)$. Así, existe correlación directa y alta entre ideación suicida y depresión, y correlaciones inversas y altas con apoyo social y reconocimiento de la homosexualidad. Adicionalmente, en la Tabla 2 se pueden observar las correlaciones entre la ideación suicida (total y por dimensiones) y los diferentes factores de las pruebas de CES-D, AS y RH. Con esto, se podría afirmar que, mientras la depresión en 
sus diferentes dimensiones es un factor de riesgo para la ideación suicida, el reconocimiento de la homosexualidad y el apoyo social son factores de protección.

\section{Tabla 2.}

Correlación entre los componentes de las variables de estudio

\begin{tabular}{llccc}
\hline \multirow{2}{*}{ Escala } & \multicolumn{1}{c}{ Factor } & $\begin{array}{c}\text { Total } \\
\text { PANSI }\end{array}$ & $\begin{array}{c}\text { Is } \\
\text { Negativa }\end{array}$ & $\begin{array}{c}\text { IS } \\
\text { Positiva }\end{array}$ \\
\hline \multirow{2}{*}{ ces-d-r } & Afecto depresivo & .616 & .562 & .518 \\
& Aspectos sociales & .644 & .513 & .609 \\
& Síntomas somáticos & .581 & .507 & .526 \\
& Afecto positivo & .711 & .593 & .662 \\
& Depresión Total & .744 & .638 & .669 \\
\multirow{2}{*}{ AS } & Apoyo Social & -.626 & -.580 & -.584 \\
& Reconocimiento Personal & -.508 & -.527 & -.429 \\
& Reconocimiento Social & -.453 & -.483 & -.385 \\
& RH Total & -.515 & -.541 & -.436 \\
\hline
\end{tabular}

Nota: En todos los casos $\mathrm{p}<0.001 ; \mathrm{P}>0.978$.

\section{Discusión}

El presente trabajo buscó determinar la relación existente entre apoyo social, depresión y reconocimiento de la homosexualidad con la ideación suicida, encontrando correlaciones inversas entre apoyo social e ideación suicida y entre reconocimiento de la homosexualidad e ideación suicida; y correlaciones directas entre depresión e ideación suicida.

En primer lugar, en comparación con los datos obtenidos de población general, se encontró que los participantes tienen mayores puntuaciones de ideación suicida total e ideación suicida negativa. Al respecto, varios estudios han demostrado que la conducta suicida es más frecuente entre las poblaciones LGBTI+ que en la población heterosexual (Madrigal y Suárez, 2000; Osma, 2004; Ortiz y García, 2005; Schlatter, Irala y Escamilla, 2005; Granados y Delgado, 2008; Langhinrichsen, Lamis y Malone, 2010; Costa, Pereira y Leal, 
[10] Ana Karen Ceballos Mora, Christian Alexander Zambrano Guerrero, Fredy Hernán Villalobos Galvis, Sara Natalia Guerrero Caicedo

2013; Irwin y Austin, 2013; Woodward, Pantalone y Bradford, 2013; Barros dos Santos et al., 2017; Avendaño et al., 2019). En coherencia con lo anterior, se plantea que el aumento de la ideación y de la conducta suicida en las minorías sexuales está relacionado con factores específicos de estrés tales como: reducido apoyo social, depresión, ocultamiento, entre otros (Langhinrichsen, Lamis y Malone, 2010; Irwin y Austin, 2013; Costa, Pereira y Leal, 2013), lo que concuerda con la relación significativa entre la minoría sexual y las altas tasas de suicidio reportadas en el trabajo de Mereish, O’Cleirigh y Bradford (2013).

Asimismo, en este estudio se halló que los participantes presentan mayores puntuaciones de depresión y menores puntuaciones de apoyo social que la población general, hallazgo que guarda correspondencia con otras investigaciones que han demostrado que la población LGBTI+ está en mayor riesgo que la heterosexual de presentar afectaciones en la salud mental como depresión, suicidio e ideación e intentos suicidas, además de problemas como la auto aceptación de la orientación sexual (Shenkman y Shmotkin, 2010; Zambrano, Ceballos y Ojeda, 2017; Zambrano, Hernández y Guerrero, 2019).

Por otro lado, se presentó una relación directa entre depresión e ideación suicida, lo cual indica que las personas homosexuales que obtuvieron puntuaciones significativas de depresión tienen gran riesgo de presentar ideación suicida. Langhinrichsen, Lamis y Malon (2010) e Irwin y Austin (2013) encontraron que tanto la desesperanza como la depresión funcionan como mediadores importantes de la propensión al suicidio en homosexuales y bisexuales; asimismo, Avendaño et al. (2019) muestran que la desesperanza se relaciona con la ideación suicida. Al respecto, Shenkman y Shmotkin (2010) refieren que mayores síntomas depresivos son más frecuentes entre lesbianas y gais en comparación con heterosexuales, debido a la exposición al abuso verbal y físico, la actitud de acoso de sus compañeros y familiares, las tasas relativamente altas de abuso sexual y las dificultades para auto aceptar su orientación sexual.

Los resultados indicaron además una relación inversa entre apoyo social e ideación suicida, similar a lo hallado por Sivasubramanian et al. (2011) quienes evidencian el papel protector proporcionado por el apoyo social percibido 
en los participantes homosexuales, ya que quienes tienen niveles más altos de apoyo social de la familia y amigos, presentan menor riesgo de ideación, intento suicida o depresión mayor. El apoyo social puede jugar un papel importante en la comprensión de la relación entre personas del mismo sexo y la propensión del suicidio, puesto que las relaciones de apoyo con padres, amigos y compañeros pueden promover el sano desarrollo y reducir el riesgo de psicopatologías. La presencia de una red de apoyo social puede funcionar como un amortiguador en la lucha contra la sensación de aislamiento y de discriminación, que pueden hacer que el suicidio sea considerado por los homosexuales como la única solución viable ante los problemas que presentan (Langhinrichsen, Lamis y Malone, 2010).

Nuestros resultados coinciden además con los presentados por Wilson, Zeng y Blackburn (2011), Langhinrichsen, Lamis y Malone (2010) e Irwin y Austin (2013), quienes manifiestan que la falta de apoyo social de pares y familia es directamente proporcional a la conducta suicida, mientras que mayores niveles de apoyo social de familiares y amigos se asociaron con una disminución de probabilidades de ideación suicida. De manera complementaria, hay una asociación entre el rechazo de los padres a causa de la orientación sexual y un mayor riesgo de intentos de suicidio entre jóvenes homosexuales; concretamente, personas que experimentaron rechazos frecuentes de sus padres o cuidadores durante la adolescencia, fueron ocho veces más propensas a reportar un intento de suicidio que aquellas cuyos padres aceptaron su orientación sexual homosexual (Haas et al., 2011). En resumen, el apoyo social es un factor protector frente al suicidio en las personas homosexuales, puesto que ayudaría a enfrentar las diversas situaciones adversas que viven quienes visibilizan su preferencia homosexual (Wilson, Zeng y Blackburn, 2011; Button, O'Connell y Gealt, 2012).

Por otra parte, en este estudio el reconocimiento de la homosexualidad tuvo una relación inversa con la ideación suicida, por lo que, a menor reconocimiento de la homosexualidad, mayores puntuaciones de ideación suicida. Se entiende que el reconocimiento de la homosexualidad es el proceso en el que el individuo acepta sus deseos y sentimientos, proyecta libremente su sexualidad y es legitimado por la sociedad como una persona homosexual 
[12] Ana Karen Ceballos Mora, Christian Alexander Zambrano Guerrero, Fredy Hernán Villalobos Galvis, Sara Natalia Guerrero Caicedo

(Díaz, 2004). Alcanzar el reconocimiento de la orientación sexual homosexual implica enfrentar los discursos sociales sobre la homosexualidad que generan miedo, culpa y rechazo, que promueven actitudes discriminatorias como la violencia homofóbica, la cual reproduce las valoraciones negativas de la homosexualidad y vulnera la salud mental de los homosexuales, configurando un estado de estrés negativo (distress), que hace que las personas sean más susceptibles a trastornos mentales y altos porcentajes de conducta suicida (Granados y Delgado, 2008).

Un factor significativo que contribuye a los niveles elevados de intentos de suicidio entre la población LGBTI+ es el estigma social generalizado que sienten las personas que abiertamente se identifican, o son percibidos como, LGBTI+. Este estigma se manifiesta a menudo por violencia física y emocional contra ellas, así como la discriminación por parte de la familia, amigos y comunidad en general; el miedo a la violencia y la discriminación conduce a altos niveles de conservación en secreto de las identidades sexuales, así como a una falta de voluntad general de divulgar su identidad, que se conoce comúnmente como vivir en el closet (Halady, 2013). Cuando la orientación sexual es diferente a la heterosexual se generan conductas de rechazo en la familia, la escuela, el trabajo y los espacios públicos, dando lugar a sentimientos de miedo y desolación, reforzando las conductas de aislamiento, ocultamiento de la homosexualidad e ideación suicida (Granados y Delgado, 2008).

Si bien es cierto, la divulgación de la orientación sexual homosexual o bisexual se convierte en el principal factor de riesgo para que la persona sea víctima de violencia homofóbica y discriminación, lo cual conlleva a un incremento del riesgo de conductas suicidas (Langhinrichsen, Lamis y Malone, 2010), también es necesario afirmar que cuando las personas se reconocen como homosexuales a través de las etapas de: (a) surgimiento, (b) identificación, (c) asunción de una identidad, (d) aceptación de la identidad, (e) consolidación y (f) autoevaluación y apoyo a otros (Ardila, 2007; Maroto, 2006; Zambrano, Hernández y Guerrero, 2019), este reconocimiento personal y social de la orientación sexual es vivido como "quitarse una carga", conllevando a una condición de bienestar emocional y a un empoderamiento personal y social para enfrentar las presiones de la familia, la escuela y el entorno cercano 
y encontrar un sentido satisfactorio para su vida (Zambrano, Ceballos y Ojeda, 2017), lo cual explicaría la relación negativa hallada en este estudio, entre el reconocimiento de la orientación sexual y la ideación suicida.

Los datos obtenidos en este estudio permitirían suponer que la alta tasa de suicidios en las personas homosexuales se explicaría, en gran parte, por una reducida habilidad para buscar posibles soluciones a problemas generados por el escaso apoyo social, la depresión, un limitado reconocimiento de la orientación sexual y la presencia de ideación suicida, entonces, el principal factor de riesgo para las conductas autolíticas no sería la orientación sexual en sí misma, sino la homofobia que surge ante ella.

En ese sentido, el apoyo social y el reconocimiento de la orientación sexual por parte de familia, amigos, sociedad y Estado resultan fundamentales para minimizar los factores de riesgo y permitir que las personas homosexuales puedan construir mecanismos de afrontamiento y protección más eficaces. Por consiguiente, es importante llevar a cabo acciones que permitan que padres, maestros y comunidad en general comprendan los beneficios de brindar redes de apoyo a la comunidad con orientación sexual homosexual.

\section{Conclusiones y recomendaciones}

La ideación suicida en personas homosexuales es mayor que la de la población general, y se relaciona con las vivencias de discriminación, estigma y rechazo en su contexto familiar, laboral y social.

La depresión es considerada como un factor de riesgo frente al suicidio y en las personas homosexuales se ha encontrado que este riesgo es mayor, ya que presentan mayor propensión a vivir situaciones de conflictos internos en relación con la sexualidad, mayores niveles de victimización, aumento de la discordia familiar relacionada con la orientación sexual, aislamiento y ocultamiento.

En las personas homosexuales, el apoyo social funciona como un factor protector en la lucha contra la sensación de aislamiento y discriminación, que hacen que el suicidio sea considerado como una solución viable a los problemas que se enfrentan, puesto que las personas que se sienten apoyadas 
[14] Ana Karen Ceballos Mora, Christian Alexander Zambrano Guerrero, Fredy Hernán Villalobos Galvis, Sara Natalia Guerrero Caicedo

y acompañadas están mayormente equipadas para enfrentar situaciones de rechazo o de vulneración debido a su orientación sexual.

El reconocimiento de la homosexualidad es un factor protector frente al suicidio, puesto que cuando la persona se reconoce y acepta como homosexual puede llevar una vida sin ocultamientos y hacer frente al prejuicio, la discriminación y mensajes violentos de personas que no aceptan su orientación sexual.

Se recomienda realizar nuevas investigaciones con personas con orientación sexual homosexual en el contexto colombiano que permitan conocer la prevalencia y el desarrollo de esta problemática en dicha población. El desconocimiento de esta información puede conllevar a que las acciones de prevención e intervención frente al suicidio en personas homosexuales no cuenten con el respaldo de datos científicos que garanticen que los factores intervenidos resulten pertinentes para el manejo de este problema de salud pública.

\section{Referencias}

Ardila, R. (2007). Terapia afirmativa para homosexuales y lesbianas. Revista Colombiana de Psiquiatría, 36(1), 67-77.

Arláez, L., Hernández, G., Álvarez, D. y Cañizares, T. (1998). Conducta suicida: factores de riesgo asociados, Revista Cubana de Medicina General Integral, 14(2), 122-126.

Avendaño-Prieto, B., Betancort-Montesinos, M., Bernal-Aguirre, A., González-Martínez, L., Gómez-Sánchez, S. y Villalobos-Sánchez, C. (2019). Celos, desesperanza e ideación suicida en población con orientación sexual diversa. Universitas Psychologica, 18(4), 1-12.

Bagley, C. y Tremblay, P. (1997). Suicidal behaviors in homosexual and bisexual males. Crisis: The Journal of Crisis Intervention and Suicide Prevention, 18(1), 24-34. doi: https://doi.org/10.1027/0227-5910.18.1.24

Benavides, K., Villota, N. y Villalobos, F. (2019) Conducta suicida en Colombia: una revisión sistemática. Revista de Psicopatología y Psicología Clínica, 24, 181-195.

Barros Dos Santos, H., Reschetti, S., Martínez, M., Nunes, N. y Cabral de Paulo, C. (2017). Factores asociados a la presencia de ideación suicida entre universitarios. Revista Latino-Americana de Enfermagem, 25, 1-8. doi: https://doi: $10.1590 / 1518-8345.1592 .2878$ 
Reconocimiento de la homosexualidad, apoyo social, depresión e ideación suicida en personas homosexuales

Button, D., O'Connell, D. y Gealt, R. (2012). Sexual Minority Youth Victimization and Social Support: The Intersection of Sexuality, Gender, Race, and Victimization. Journal of Homosexuality, 59, 18-43. doi: https://doi.org/10.1080/009 18369.2011.614903

Chaparro, P., Díaz, D. y Castañeda, C. (2019). Tendencia de la mortalidad por suicidio en las áreas urbanas y rurales de Colombia, 1979-2014. Biomédica: Revista del Instituto Nacional de Salud, 39(2), 339-353. doi: https://doi.org/10.7705/ biomedica.v39i3.4427.

Chomali, F. (2008). Algunas consideraciones para el debate actual acerca de la homosexualidad. Santiago: Universidad Católica de Chile.

Cochran, S. y Mays, V. (2009). Burden of psychiatric morbidity among lesbian, gay, and bisexual individuals in the California Quality of Life Survey. Journal of abnormal psychology, 118(3), 647-658. doi: https://doi.org/10.1037/a0016501.

Costa, P., Pereira, H. y Leal, I. (2013). Internalized Homonegativity, Disclosure, and Acceptance of Sexual Orientation in a Sample of Portuguese Gay and Bisexual Men, and Lesbian and Bisexual Women. Journal of Bisexuality, 13(2), 229-244.

Cortina, E., Peńa, M. y Gómez, Y. (2009). Factores psicológicos asociados a intentos de suicidio en jóvenes entre 16-25 años del Valle de Aburrá. Revista de Psicología Universidad de Antioquia, 1(1), 55-74.

Díaz, M. (2004). Homosexualidad y Género. Cuicuilco Nueva Época, 11(31), 1-12.

Faul, F., Erdfelder, E., Lang, A. y Buchner, A. (2007). G*Power 3: A flexible statistical power analysis program for the social, behavioral, and biomedical sciences. Behavior Research Methods, 39, 175-191. doi: https://doi.org/10.3758/ BF03193146.

González, C. y Andrade, P. (1995). La relación de los hijos con sus progenitores y sus recursos de apoyo: correlación con la sintomatología depresiva y la ideación suicida en los adolescentes mexicanos. Salud Mental, 18, 41-48.

G*Power 3.1 manual (2020). Descargado el 12, diciembre, 2020 de https://www. psychologie.hhu.de/fileadmin/redaktion/Fakultaeten/Mathematisch-Naturwissenschaftliche_Fakultaet/Psychologie/AAP/gpower/GPowerManual.pdf.

Granados, J. y Delgado, G. (2008). Identidad y riesgos para la salud mental de jóvenes gays en México: recreando la experiencia homosexual. Cadernos de Saúde Pública, 24(5), 1042-1050.

Gutiérrez, A. Contreras, C. y Orozco, R. (2006). El suicidio. Conceptos actuales. Salud Mental, 29(5), 66-74. 
[16] Ana Karen Ceballos Mora, Christian Alexander Zambrano Guerrero, Fredy Hernán Villalobos Galvis, Sara Natalia Guerrero Caicedo

Haas, A., Eliason, M., Mays, V., Mathy, R., Cochran, S., D’Augelli, A., ..., Clayton, P. (2011). Suicide and Suicide Risk in Lesbian, Gay, Bisexual, and Transgender Populations: Review and Recommendations. Journal of Homosexuality, 58, $10-51$.

Halady, S. (2013). Attempted Suicide, LGBT Identity, and Heightened Scrutiny. The American Journal of Bioethics, 13(3), 20-22. doi: https://doi.org/10.1080/1526 5161.2012.760676

Iraurgi, I. (2009) Evaluación de resultados clínicos (II): las medidas de la significación clínica o los tamaños del efecto. Norte de Salud Mental, 34, 94-110.

Irwin, J. y Austin, E. (2013). Suicide Ideation and Suicide Attempts Among White Southern Lesbians, Journal of Gay y Lesbian Mental Health, 17(1), 4-20. doi: https://doi.org/10.1080/19359705.2012.711552

Jasso, R. (2013) La dimensión espacial del suicidio y su vínculo con el mercado laboral mexicano (2000-2004). Revista de la Facultad de Ciencias Económicas: Investigación y Reflexión, XXI(1), 189-216.

Langhinrichsen, J., Lamis D. y Malone, P. (2010). Sexual Attraction Status and Adolescent Suicide Proneness: The Roles of Hopelessness, Depression, and Social Support, Journal of Homosexuality, 58(1), 52-82. doi: https://doi.org/10.1080/ 00918369.2011 .533628

Li, G., Pollitt, A. y Russell S. (2015). Depression and Sexual Orientation During Young Adulthood: Diversity Among Sexual Minority Subgroups and the Role of Gender Nonconformity. Archives of sexual behavior, 45, 697-711. doi: https://doi.org/10.1007/s10508-015-0515-3.

Madrigal, F. y Suárez, D. (2000). Suicidio en la población homosexual costarricense. CIPAC: Centro de investigación y promoción para América Central de Derechos Humanos.

Maroto, A. (2006). Homosexualidad y Trabajo Social. Herramientas para la reflexión e intervención profesional. Madrid: Siglo Xxi.

Matud, M. (1998). Investigación del estrés y su impacto en la salud de la mujer en Canarias. Informe final del proyecto financiado por el Instituto Canario de la Mujer. La Laguna (Documento inédito).

Matud, P., Carballeira, M., López, M., Marrero, R. y Ibañez, I. (2002). Apoyo Social y Salud: un análisis de género. Salud Mental, 25(2), 32-37.

Mereish, E., O'Cleirigh, C. y Bradford, J. (2013). Interrelationships between LGBTbased victimization, suicide, and substance use problems in a diverse sample of sexual and gender minorities. Psychology, Health \& Medicine, 9(1), 37-41. doi: https://dx.doi.org/10.1080\%2F13548506.2013.780129 
Reconocimiento de la homosexualidad, apoyo social, depresión e ideación suicida en personas homosexuales

Muñoz, J., Pinto, V., Callata, H., Napa, N. y Perales, A. (2006). Ideación suicida y cohesión familiar en estudiantes preuniversitarios entre 15 y 24 años, Lima 2005. Revista Peruana de Medicina y de Salud Pública, 23(4), 239-246.

Newcomb, M., Birkett, M., Corliss, H. y Mustanski, B. (2014). Sexual Orientation, Gender, and Racial Differences in Illicit Drug Use in a Sample of US High School Students. American Journal of Public Health, 104(2), 304-310. doi: https://dx.doi.org/10.2105\%2FAJPH.2013.301702.

Ojeda, E. y Villalobos, F. (2011). Elementos para una Política Pública desde la Percepción del Suicidio en Nariño. Revista Colombiana de Psicología, 20(1), 57-73.

Organización Mundial de la Salud [oms]. (2018). Suicidio. Recuperado el 18 de febrero de 2018, de: http://www.who.int/mediacentre/factsheets/fs398/es/.

Ortega, M. (2009). Empoderamiento con relación a la orientación sexual de un estudiante perteneciente a la Institución Educativa Municipal Artemio Mendoza Carvajal. Manuscrito no publicado, Universidad Mariana en Pasto, Colombia.

Ortiz, L. y García, M. (2005). Efectos de la violencia y la discriminación en la salud mental de bisexuales, lesbianas y homosexuales de la Ciudad de México. Cadernos de Saúde Pública, 21(3), 913-925.

Osma, J. (2004). Victimas de la homofobia: Consecuencias psicológicas. Cataluña: ACGIL Asociació Cristiana de Gais i Lesbianes de Catalunya.

Osman, A., Gutiérrez, P., Kopper, B., Barrios, F. y Chiros, C. (1998). The Positive and Negative Suicide Ideation Inventory: Development and Validation. Psychological Reports, 82, 783 - 793. Recuperado de: https://doi.org/10.2466/ pr0.1998.82.3.783

Peńa, M., Ortiz, Y. y Gutiérrez, I. (2009). El suicidio en Nariño: una mirada desde los observatorios del delito en cinco municipios del Departamento. Pensamiento Psicológico, 6(13), 97-108.

Plderl, M., Faistauer, G. y Fartacek, R (2010). The Contribution of School to the Feeling of Acceptance and the Risk of Suicide Attempts among Austrian Gay and Bisexual Males. Journal of Homosexuality, 57(7), 819-841. doi: https://doi. org/1\%200.1080/00918369.2010.493401

Restrepo, 1. y González, J. (2007) De Pearson a Spearman. Revista Colombiana de Ciencias Pecuarias, 20, 183-192.

Riascos, D. (2008). Etapas que caracterizan el proceso para la construcción de identidad lésbica. Manuscrito no publicado, Universidad de Nariño en Pasto, Colombia. 
[18] Ana Karen Ceballos Mora, Christian Alexander Zambrano Guerrero, Fredy Hernán Villalobos Galvis, Sara Natalia Guerrero Caicedo

Schlatter, J. Irala, J. y Escamilla, I. (2005). Psicopatología asociada a la homosexualidad. Revista de Medicina Universidad de Navarra, 49(3), 69-79.

Shenkman, G. y Shmotkin, D. (2010). Mental Health Among Israeli Homosexual Adolescents and Young Adults. Journal of Homosexuality, 58(1), 97-116.

Siabato, E. y Salamanca, Y. (2015). Factores asociados a ideación suicida en universitarios. Psychologia: avances de la disciplina, 9(1), 71-81.

Sivasubramanian, M., Mimiaga, M., Mayer, K., Anand, V., Johnson, C., Prabhugate, P. y Safre, S. (2011). Suicidality, Clinical Depression, and Anxiety Disorders Are Highly Prevalent in Men Who Have Sex with Men in Mumbai, India, A Community-recruited Sample. Psychology, Health \& Medicine, 16(4), 450-462. doi: https://dx.doi.org/10.1080\%2F13548506.2011.554645

Stone, D., Luo F., Ouyang, L., Lippy, C., Hertz, M. y Crosby, A. (2014). Sexual Orientation and Suicide Ideation, Plans, Attempts, and Medically Serious Attempts: Evidence from Local Youth Risk Behavior Surveys, 2001-2009. American Journal of Public Health, 104(2), 262-271.

Tomicic, A., Gálvez, C., Quiroz, C., Martínez, C., Fontbona, J., Rodríguez, J., Aguayo, F., Rosenbaum, C., Leyton, F. y Lagazzi, I. (2016). Suicidio en poblaciones lesbianas, gay, bisexual y trans: revisión sistemática de una década de investigación (2004-2014). Revista médica De Chile, 144(6), 723-733.

Villalobos, F. (2006). Validación de instrumentos de evaluación psicológica en estudiantes de la ciudad de San Juan de Pasto (Documento inédito)

Villalobos, F. (2009). Situación de la conducta suicida en estudiantes de colegios y universidades de San Juan de Pasto, Colombia. Salud mental, 32(2), 165-171.

Villalobos, F. (2010a). Estructura factorial y propiedades psicométricas de una escala de depresión en universitarios de Colombia. Revista Panamericana de Salud Pública 27(2), 110-116.

Villalobos, F. (2010b). Validez y fiabilidad del Inventario de Ideación Suicida Positiva y Negativa - PANSI, en estudiantes colombianos. Revista Universitas Psychologica, 9(2), 509-520.

Villalobos, F., Ojeda, E. y Luna, E. (2019). Caracterización de las conductas suicidas en adolescentes de la zona de influencia del volcán Galeras, Nariño, Colombia. Informes Psicológicos, 19(2), 163-180. doi: http://dx.doi.org/10.18566/infpsic. v19n2a011.

Vírseda, J., Amado, G., Bonilla, M. y Gurrola, G. (2011). Afrontamiento e ideación suicida en adolescentes. Revista Psicología.com, 15-16. 
Reconocimiento de la homosexualidad, apoyo social, depresión e ideación suicida en personas homosexuales

Wilson, G., Zeng, Q. y Blackburn, D. (2011). An Examination of Parental Attachments, Parental Detachments and Self-Esteem across Hetero-, Bi-, and Homosexual Individuals. Journal of Bisexuality, 11(1), 86-97. doi: https://doi.org/10. 1080/15299716.2011.545312

Woodward, E., Pantalone, D. y Bradford, J. (2013). Differential Reports of Suicidal Ideation and Attempts of Questioning Adults Compared to Heterosexual, Lesbian, Gay, and Bisexual Individuals, Journal of Gay y Lesbian Mental Health, $17(3), 278-293$.

Zambrano, C., Hernández, P. y Guerrero, A. (2019). Proceso de reconocimiento de la orientación sexual homosexual en estudiantes de una universidad pública. Psicogente, 22(41), 1-29. doi: https://doi.org/10.17081/psico.22.41.3310

Zambrano, C., Ceballos, A. y Ojeda, D. (2017). Reconocimiento de la orientación sexual homosexual. Revista Psicoespacios, 11(19), 77-93. 